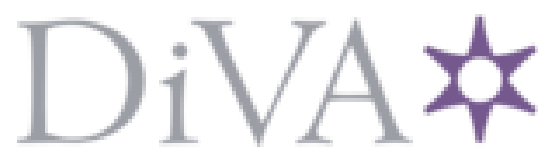

http://www.diva-portal.org

This is the published version of a paper published in Plastics, rubber and composites.

Citation for the original published paper (version of record):

Kaufmann, M., Czumanski, T., Zenkert, D. (2009)

Manufacturing process adaptation for integrated cost/weight optimisation of aircraft structures.

Plastics, rubber and composites, 38(2): 162-166(5)

http://dx.doi.org/10.1179/174328909X387793

Access to the published version may require subscription.

N.B. When citing this work, cite the original published paper.

Permanent link to this version:

http://urn.kb.se/resolve?urn=urn:nbn:se:kth:diva-11474 


\title{
Manufacturing process adaptation for integrated cost/weight optimisation of aircraft structures
}

\author{
Markus Kaufmann ${ }^{a}$, Thomas Czumanski ${ }^{b}$ and Dan Zenkert ${ }^{a}$ \\ ${ }^{a}$ Kungliga Tekniska Högskolan (KTH), 10044 Stockholm, Sweden \\ ${ }^{b}$ Audi AG, Industrial Engineering and Operations Management, \\ 74148 Neckarsulm, Germany
}

March 2009

\begin{abstract}
A methodology is developed that enables cost-efficient design of composite aircraft structures. In earlier work, a cost/weight optimisation framework was presented. This framework is here enhanced by a module that minimises the manufacturing cost in each iteration by adaptation of manufacturing parameters. The proposed framework is modular and applicable to a variety of parts and geometries. Commercially available software is used in all steps of the optimisation. The framework extension is added to an existing cost/weight optimisation implementation and tested on an airliner centre wing box rear spar. Three optimisation runs are performed, and a low cost, an intermediate and a low weight design solution are found. The difference between the two extreme solutions is $4.4 \%$ in manufacturing cost and $9.7 \%$ in weight. Based on these optimisation trials, the effect of the introduced parameter adaptation module is analysed.
\end{abstract}

Keywords: Cost/weight optimisation, Process adjustment, Parameter adaptation, Aircraft structures

\section{Introduction}

As the cost for jet fuel rises, the airliner's fuel consumption has to be reduced in order to offer a competitive product in terms of overall operating cost. One way to cut the fuel consumption is to lower the gross weight, thus increasing the performance of the aircraft structure. Composite materials can lower the structural weight significantly. The drawback, however, is the increased manufacturing cost. The minimum weight and the minimum cost solutions are two extremes that often 
contradict each other. Therefore an optimal design solution is a tradeoff between the manufacturing cost, structural requirements, process capabilities and the materials available. A measure of the quality of a design solution is the direct operating cost (DOC) where the cost of flight, depreciation, maintenance, etc. are combined in a single parameter.

Kelly et al. [1], Wang et al. [2], Curran et al. [3,4] and Kaufmann et al. [5] have incorporated weight penalty approaches for the optimisation of aircraft structures. These approaches had in common that the objective function is formed by weighted sums, the latter containing the manufacturing cost and the weight of the part. In particular, Kaufmann et al. proposed a simplified direct operating cost as the objective function. It contained the manufacturing cost and the life fuel burn cost as geometry-driven attributes of aircraft components. Other costs that appeared during the lifecycle of the aircraft [6] such as cabin cost, navigation cost, maintenance and repair, and dismantling costs were neglected. The proposed objective function can be written as

$$
D O C=C_{m a n}+p W
$$

The manufacturing cost $C_{\text {man }}$ includes all operations to produce the part, from tooling to surface finish along with associated labour and material costs. It also includes costs for assembly. The second component, the cost of fuel burn, is related to the mass of the part and could therefore be expressed using the lifetime fuel burn cost per unit aircraft mass $p$ set against the computable part weight $W$. Additionally, any candidate design must fulfil the structural requirements of the specified part while aiming for the $D O C$ minimum, leading to a formulation of the optimisation problem as

$\begin{aligned} \min & D O C \\ \text { subject to } & \text { structural requirements } \\ & \underline{x}_{i}<x_{i}<\bar{x}_{i}, \quad i=1 \ldots n,\end{aligned}$

where $x_{i}$ are variables, and $\underline{x}_{i}$ and $\bar{x}_{i}$ are their lower and upper limits, respectively. The weight penalty $p$ may have a decisive impact on where the cost/weight minimum is located. Wang et al. proposed a value of $\$ 500 / \mathrm{kg}$, whereas comparable values of Curran et al. tended to be much lower $(\$ 86-150 / \mathrm{kg})$. Vogelesang and Gunnink [7] quoted a cost reduction of $€ 19.2$ to $€ 435$ per year per $\mathrm{kg}$ weight saving. On a life span of approximately 25 years, this value would accumulate to $€ 481$ to $€ 1087$ per $\mathrm{kg}$ saved weight. Eventually, an own estimation was performed based on current fuel prices and fuel consumptions of an A330 which resulted in $€ 1500 / \mathrm{kg}$ to $€ 2000 / \mathrm{kg}$ (personal communication with Olle Björk from Scandinavian Airlines, Sweden, April 2007).

The optimisation problem given in Equation (2) was applicable to arbitrary components of aircraft structures, such as the skin/stringer element presented in previous work [5]. There, the authors concluded that a major drawback of the utilised approach was the locked manufacturing processes which needed to be declared 
beforehand and remain the same during every iteration of the optimisation process. The stiffener geometry, for example, could be manufactured in different ways. The choice of tools (cutter material and size) had an influence on the manufacturing costs and should thus not be kept unaltered during optimisation. Therefore, the process conditions or parameters such as tool specifications, machine properties or even inspection methods should be incorporated in order to achieve the lowest possible manufacturing cost for a given geometry. Such a parameter adaptation is illustrated in Figure 1.

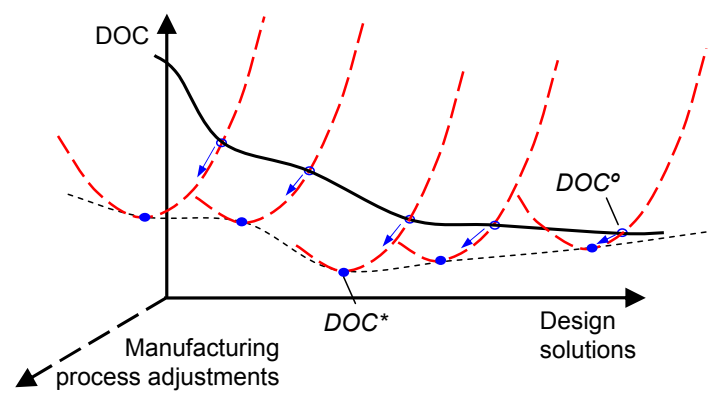

Figure 1: Minimisation of direct operation cost is schematically illustrated: $D O C^{*}$ and $D O C^{\circ}$ refer to optimal direct operating cost with and without manufacturing process adjustments, respectively.

The solid line shows the objective function value depending on feasible design solutions, without adapting the manufacturing process to these particular solutions. The dashed line illustrates the optimised $D O C$ values through process adaptations, whereas each of the five parabolic curves illustrates the scope of process adjustments for a particular design solution. The optimisation potential for each design is indicated by the arrows and points, respectively.

\section{Method}

The optimisation framework proposed by Kaufmann et al. [5] was used to optimise the design of skin/stringer elements by linking a CAD model (ABAQUS/CAE), a manufacturing cost model (SEER-MFG) and results from finite element analysis of the component as illustrated in Figure 2. For further information on these tools see the documentation $[8,9]$ and http://www.galorath.com. In order to automate the cost calculation, the SEER-MFG server mode was used for the manufacturing cost modelling. The skin/stringer element cost was modelled once and the information needed to build that model was exported in the form of a command file. In every iteration, this file was then altered according to the variable changes made by the solver. Thereafter, the manufacturing cost $C_{\text {man }}$ was calculated and fed back. The finite element analysis for each given design solution was carried out with ABAQUS/CAE, where the structural model was parameterised by means of Python 
scripts. The solver synchronised the two parts of the loop and controlled the overall process. A gradient-based optimisation method (the method of moving asymptotes, see Svanberg [10,11] and Bruyneel et al. [12]) was chosen for that purpose, by means of the optimisation code Xopt [9].

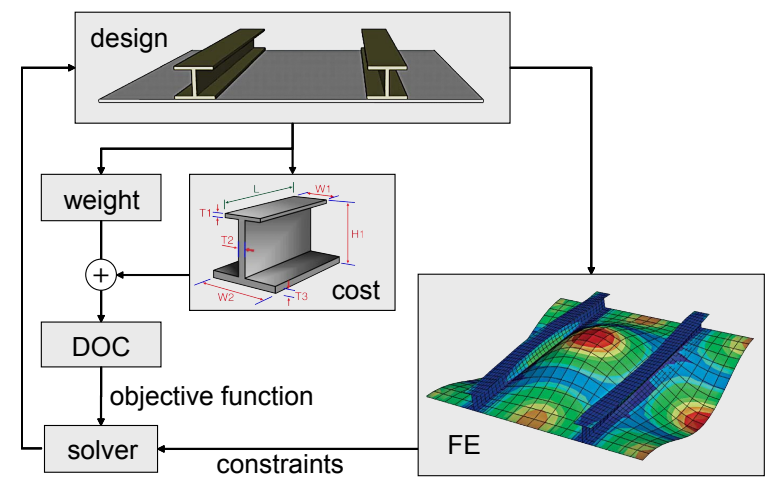

Figure 2: Optimisation loop including a design variable database, an ABAQUS FE model, a SEER-MFG cost model, a parameterised weight model and the Xopt solver.

\subsection{Framework extension}

The idea of the parameter adaptation was incorporated in the existing framework. For this task, a subroutine was integrated into the scheme described, optimising the process within SEER-MFG while keeping the geometrical design, defined by the variables $x_{i}$, unchanged. For the incorporation of process knowledge and the retrieval of appropriate process options, facts and rules had to be stored in the form of a knowledge base. A second solver was needed to process the design information and the stored knowledge in order to manipulate the cost model accordingly.

The extended optimisation framework is depicted in Figure 3. The overall architecture can be seen as a two level optimisation methodology (see Gantois and Morris [13]) altering the part design in the top level routine and optimising process parameters in a lower level routine, the latter being termed process adaptation module (PAM). The process adaptation module was implemented in Matlab.

\section{Case study}

Because of the high complexity of the optimisation routine, it was decided to show the effect of PAM by optimising only two selected process features on a rear spar of an airliner's centre wing box. Note that input data and results are purely academic and not related to any existent structure. 


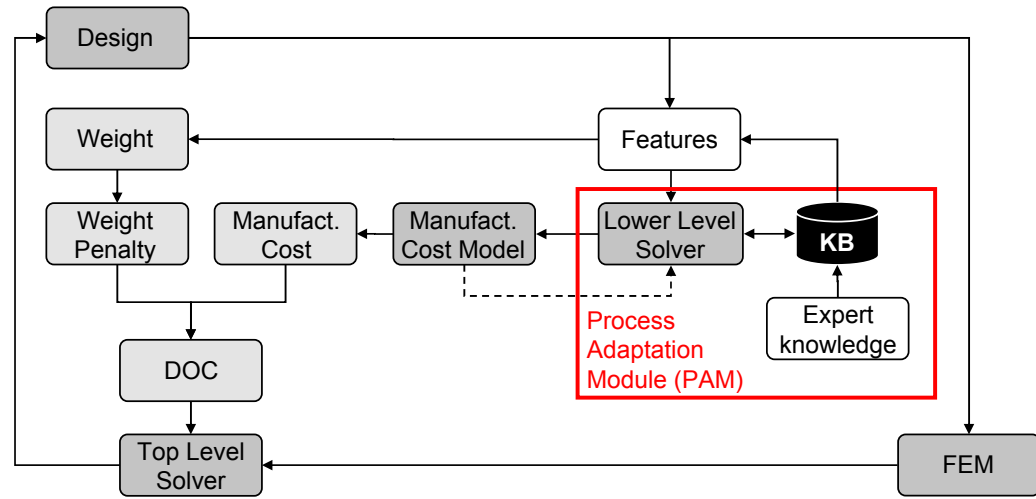

Figure 3: Optimisation framework extended by PAM which containing knowledge base and lower level solver.

\subsection{Part design, geometry and materials}

The design of the centre wing box (CWB) rear spar is shown in Figure 4. The web of the spar can be separated into three sections: the "rib 1" section, the "far field" section and the "rib 6" section. This division was done in order to enable the layup's thicknesses and fibre distributions to differ in each section. The upper and lower edges were flanged for the attachment of the upper and lower CWB cover. Four vertical I-shaped stiffeners were attached to the outer side of the web and bolted to eight fittings at the inner side, the latter serving as the connection to the struts in the CWB. Additionally, a manhole was positioned between the third and the fourth stiffener. Some geometric parameters were fixed, as shown in Table 1. No other geometric data were predefined and could potentially serve as design variables for the cost/weight optimisation. Two different materials were used for the main components. The web was made of the composite laminate M21/T800, whereas the vertical stiffeners and the internal fittings were made of aluminium alloy 7010 . The bolts used to fasten the stiffeners to the internal fittings were made of Ti6 Al- $4 \mathrm{~V}$.

\subsection{Load cases}

The FE model was tested using two load cases:

- an internal pressure load case (due to fuel overfilling)

- a load case derived from the cabin pressure.

Other test cases, such as turbulence (maximum upbend) and manoeuvre (maximum shear and downbend) were neglected since they were regarded as non-critical. 


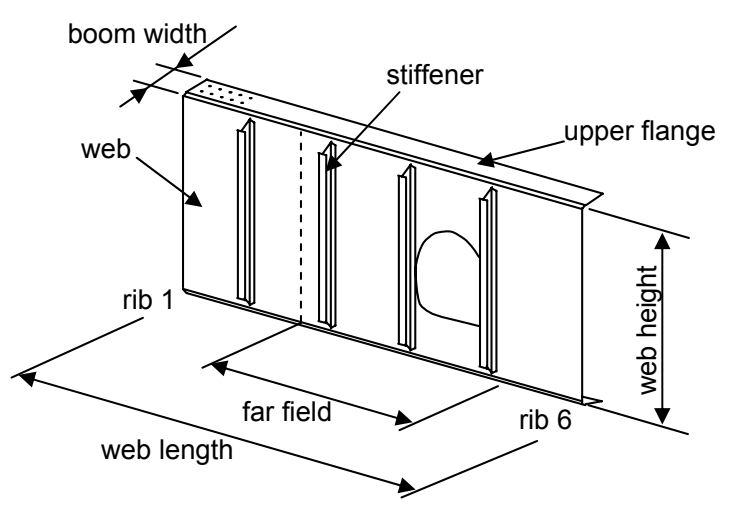

Figure 4: Outer side of CWB rear spar

Table 1: Baseline geometry data for CWB rear spar

\begin{tabular}{lr}
\hline Geometric parameters & Value \\
\hline web length & $2500 \mathrm{~mm}$ \\
web height & $909 \mathrm{~mm}$ \\
boom width & $300 \mathrm{~mm}$ \\
stringer length & $809 \mathrm{~mm}$ \\
stringer foot thickness & $6 \mathrm{~mm}$ \\
stringer distance & $503 \mathrm{~mm}$ \\
rib 1 section length & $500 \mathrm{~mm}$ \\
far field section length & $1600 \mathrm{~mm}$ \\
rib 6 section length & $400 \mathrm{~mm}$ \\
bolt diameter & $6.35 \mathrm{~mm}$ \\
ply thickness & $0.26 \mathrm{~mm}$ \\
\hline
\end{tabular}

\subsection{Parameterisation of the rear spar}

The optimisation procedure required the parameterisation of the structural model in terms of design variables $x_{i}$. Variables of particular interest were the thickness of the spar and the geometry of the I-shaped stiffeners. Both components had major impact on the spar strength and stiffness. Parameters that had a predictable influence on the $D O C$ were not selected as variables. The internal fittings, for instance, did not contribute much to the spar stiffness, strength and weight. Therefore they remain unchanged. The optimisation was enhanced by the division of the web into three sections with separate different thicknesses variables, i.e. each section possibly having a different layup. Hence, a parameterisation with 13 design variables was chosen as described in Table 2 and in Figure 5. 
Table 2: Design variables for CWB rear spar

\begin{tabular}{lll}
\hline$x_{i}$ & Description & Location \\
\hline$x_{1}$ & ply thickness in $0^{\circ}$ & rib 1 section \\
$x_{2}$ & ply thickness in $45^{\circ}$ & rib 1 section \\
$x_{3}$ & ply thickness in $90^{\circ}$ & rib 1 section \\
$x_{4}$ & ply thickness in $0^{\circ}$ & far field section \\
$x_{5}$ & ply thickness in $45^{\circ}$ & far field section \\
$x_{6}$ & ply thickness in $90^{\circ}$ & far field section \\
$x_{7}$ & ply thickness in $0^{\circ}$ & rib 6 section \\
$x_{8}$ & ply thickness in $45^{\circ}$ & rib 6 section \\
$x_{9}$ & ply thickness in $90^{\circ}$ & rib 6 section \\
$x_{10}$ & stringer height & \\
$x_{11}$ & stringer width & \\
$x_{12}$ & stringer flange thickness & \\
$x_{13}$ & stringer pocket depth & \\
\hline
\end{tabular}

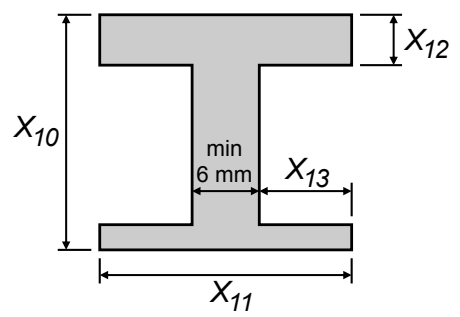

Figure 5: Parameterisation of the I-type stringer

The constraints regarding the web layup (design variables $x_{1}$ to $x_{9}$ ) implied that the minimum thickness in each direction was at least two plies. The lower limit for the stringer height $\left(x_{10}\right)$ and the upper limit for the stringer flange thickness $\left(x_{12}\right)$ were adjusted in a way that the pocket width was always larger than the minimum cutter diameter $(12 \mathrm{~mm})$, illustrating how constraints from manufacturing could be included in the optimisation problem. Regarding the pocket depth $\left(x_{13}\right)$ a lower limit of $10 \mathrm{~mm}$ was chosen for assembly reasons (6.35 $\mathrm{mm}$ fastener diameter). Finally, a constraint was included to avoid negative values for the pocket depth $\left(x_{13}\right)$, while maintaining a minimum stringer web thickness of $6 \mathrm{~mm}$. The optimisation problem could then be rewritten as

$$
\begin{array}{ll}
\min _{x} & C_{\text {man }}^{\text {opt }}(x, y) \text { where y solves } \\
\min _{x \mid y} & C_{\text {man }}^{\text {opt }}(x, y)
\end{array}
$$




$$
\begin{array}{ll}
\text { subject to } & \text { prescribed load case } \\
& 0.52<x_{i}<10.00 \quad x_{i}=1 \ldots 9 \\
& 34.00<x_{10}<100.00 \\
& 25.00<x_{11}<100.00 \\
& 2.00<x_{12}<16.00 \\
& 10.00<x_{13}<47.00 \\
& x_{13}<\left(x_{11}-6\right) / 2
\end{array}
$$

The selected process features for parameter adjustments $y$ were two parameters for the pocket milling operations of the stiffeners, specifically the cutter diameter $y_{1}$ and the cutting tool material $y_{2}$.

\section{Results}

In the following, three optimisation test runs are analysed. The values for manufacturing cost and part weight are briefly addressed, followed by observations regarding the effects on the stringer milling process and the web layup.

\subsection{Manufacturing cost and weight}

The total manufacturing cost and the part weight for the found optima are given in Table 3, where the weight penalty $p$ (measured in $€$ per $\mathrm{kg}$ ) was set to 0 in the 'min Cost' test run, to $€ 1500 / \mathrm{kg}$ in the test run named 'WP1500' and to infinity in the 'min Weight' test run. The initial values of the 13 design variables were the same in each test run.

As could be expected, the manufacturing cost was the highest in the 'min Weight' test run and the lowest in the 'min Cost' test run. The difference in manufacturing cost was about $4.4 \%$. A similar trend could be seen for the 'min Weight' run, resulting in the smallest manufacturing weight, followed by 'WP1500' and 'min Cost'. The difference in weight was about $9.7 \%$ for the two opposing solutions. The optimum design solution of 'WP1500' was examined in SEER-MFG and the total manufacturing cost of the optimum design for the 'WP1500' test run was allocated with labour, material, tooling and other costs, as illustrated in Figure 6.

Table 3: Optimum values of test runs for cost/weight optimisation of CWB rear spar, using the extended optimisation framework

\begin{tabular}{lrrr}
\hline Test run & min Cost & WP1500 & min Weight \\
\hline Manufacturing cost $[€]$ & 14996 & 15203 & 15657 \\
Weight [kg] & 76.89 & 70.59 & 70.10 \\
\hline
\end{tabular}




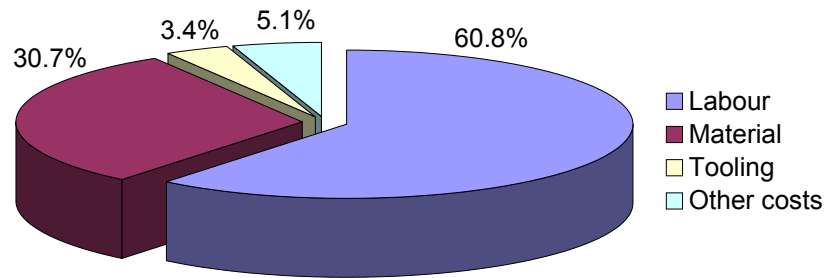

Figure 6: Cost allocation for CWB rear spar at 'WP1500' test run optimum. More than $60 \%$ is related to labour (machining, prepreg layup, debulking, etc.), offering potential for cost reductions by process adjustments

\subsection{Stringer milling process}

Detailed results regarding the stringer shape are shown in Table 4. As can be seen, the stringer height and the pocket width increased with increasing weight penalty, whereas the stringer width and the pocket depth were constant for high weight penalties. Regarding the process adaptations made by PAM, it was notable that the cutter diameter changed with the stringer geometry. In the present cases, the optimum cutter diameter was the one closest below the pocket height, i.e. a $12 \mathrm{~mm}$ cutter for a pocket height of $14.22 \mathrm{~mm}$ and a $32 \mathrm{~mm}$ cutter for a pocket height of 60.79 and $81.43 \mathrm{~mm}$ respectively. The cutting tool material was carbide for all design solutions, as high speed steel did not lead to a reduction in milling cost.

In order to analyse the effect of PAM, the cutter diameter and the cutting tool material were set to values feasible for any design solution (12 mm, high speed steel) in the optimum SEER-MFG model for the 'WP1500' test run. A closer look to the stringer manufacturing cost revealed a reduction of $30 \%$ through the application of PAM. The share of this cost with respect to the total cost was $4.3 \%$ in that particular case. It is noteworthy that this reduction was achieved by optimising only two process parameters, thereby hinting an even higher cost saving potential for more complex aluminium structures.

Table 4: Selected parameters of stringer geometry and pocket milling operations according to optimum design solutions

\begin{tabular}{lrrr}
\hline Test run & min Cost & WP1500 & min Weight \\
\hline stringer height $[\mathrm{mm}]$ & 34.00 & 69.61 & 89.43 \\
pocket width $[\mathrm{mm}]$ & 14.22 & 60.79 & 81.43 \\
pocket depth $[\mathrm{mm}]$ & 10.00 & 46.17 & 46.11 \\
cutter diameter $[\mathrm{mm}]$ & 12 & 32 & 32 \\
cutting tool & $\mathrm{C}$ & $\mathrm{C}$ & $\mathrm{C}$ \\
\hline
\end{tabular}




\subsection{Web layup}

The optimum data obtained for the web layup are given in Table 5. Note the change in the Rib 1 section, where the thickness of the web was reduced by $44 \%$ in favour of stockier stiffeners.

Table 5: Thicknesses of three web sections and corresponding weighted average values for obtained optimum designs

\begin{tabular}{lrrr}
\hline Test run & min Cost & WP1500 & min Weight \\
\hline rib 1 section [mm] & 9.36 & 5.22 & 5.51 \\
far field section $[\mathrm{mm}]$ & 13.64 & 14.16 & 13.71 \\
rib 6 section [mm] & 16.31 & 14.02 & 14.82 \\
\hline
\end{tabular}

\section{Conclusions}

The amount of data obtained with these test runs was not sufficient to draw reliable and detailed conclusions. Therefore, the following items are of rather general nature. Beside the validation of the functionality of the extended framework, the test results given above indicate that:

1. the process adjustments made by PAM can lead to a significant reduction of stringer manufacturing cost

2. more process adjustments need to be optimised to achieve a significant reduction of the total manufacturing cost

3. problems with local minima may arise during the optimisation

The model approach was formulated as an optimisation problem which incorporated the manufacturing cost and the part weight in the objective function, subject to structural requirements and design variables. The PAM was a first step towards the sub-optimisation of manufacturing processes. It provided the most cost-efficient set of cutter diameter and cutting tool material to be chosen for the machining of the aluminium stringers.

The integration of PAM into the existing framework significantly extended the calculation time of an optimisation run. Another special tool or an enhancement of Xopt is therefore needed to reduce the number of invocations of SEER-MFG in the lower level optimisation. Nevertheless, first test runs have shown the functionality of the integrated framework with an upper level routine (Xopt) and a lower level routine (PAM) for cost optimisation of the CWB rear spar. Additionally, PAM was developed using a modular script structure and may therefore be easily extended or included in other structural optimisation problems that incorporate SEER-MFG. If tailored to parts for which geometry and manufacturing processes are not predetermined, it may have a major impact. 


\section{Future work}

A way for reducing computing time of PAM should be found, especially when more process features are to be processed. It should further be modified and tailored to the optimisation of other parts and manufacturing methods, with a special focus on composite structures, e.g. by its application to the optimisation of draped prepreg materials. Regarding the cost model, a module for the estimation of maintenance, repair and overhaul cost with respect to composite parts could be developed.

\section{Acknowledgments}

This work is part of the European Framework Program 6, project ALCAS, AIP4-CT2003-516092. Special thanks go to Alfgam AB for the use of Xopt and to Galorath International for the use of the cost estimation package SEER-MFG.

\section{Bibliography}

[1] D. Kelly, K. Wang, and S. Dutton. A guided tradeoff for cost and weight for generating optimal conceptual designs. In Collection of Technical Papers AIAA/ASME/ASCE/AHS/ASC Structures, Structural Dynamics and Materials Conference, volume 2, pages 894-904, 2003.

[2] K. Wang, D. Kelly, and S. Dutton. Multi-objective optimisation of composite aerospace structures. Composite Structures, 57(1):141-148, 2002.

[3] R. Curran, S. Raghunathan, and M. Price. Review of aerospace engineering cost modelling: The genetic causal approach. Progress in Aerospace Sciences, 40(8):487-534, 2004 .

[4] R. Curran, A. Rothwell, and S. Castagne. Numerical method for cost-weight optimization of stringer-skin panels. Journal of Aircraft, 43(1):264-274, 2006.

[5] M. Kaufmann, D. Zenkert, and P. Wennhage. Integrated cost/weight optimization of composite skin/stringer elements. In Takashi Ishikawa, editor, Proceedings of 16th International Conference on Composite Materials, Kyoto, 2007.

[6] J. Roskam. Airplane Design Part VIII: Airplane Cost Estimation Design Development and Manufacturing and Operating. Darcorporation, 1990.

[7] L. B. Vogelesang and J. W. Gunnink. Arall: A materials challenge for the next generation of aircraft. Materials and Design, 7(6):287-300, 1986.

[8] ABAQUS. Online Documentation. ABAQUS, Inc., March 2006. Version 6.6-1.

[9] Xopt. Optimeringsprogrammet Xopt - Manual. Alfgam Optimering AB, 2.0 edition, October 2001.

[10] K. Svanberg. Method of moving asymptotes - a new method for structural optimization. Journal for Numerical Methods in Engineering, 24(2):359-373, 1987. 
[11] K. Svanberg. A globally convergent version of MMA without linesearch. In Proceedings of the First World Congress of Structural and Multidisciplinary Optimization, Goslar (Germany), pages 9-16, May/June 1995.

[12] M. Bruyneel, P. Duysinx, and C. Fleury. A family of MMA approximations for strutural optimization. Structural and Multidisciplinary Optimization, 2002.

[13] K. Gantois and A. J. Morris. The multi-disciplinary design of a large-scale civil aircraft wing taking account of manufacturing costs. Structural and Multidisciplinary Optimization, 28:31-46, 2004. 